\title{
Horizontal Expansion of Eruptive Prominences
}

\author{
Bogdan Rompolt \\ Astronomical Institute of Wroctaw University, 51-622 Wroctaw, ul. \\ Kopernika 11, Poland
}

\section{Introduction}

It is well known that quiescent prominences (QPs) as well as active region prominences (ARPs) occasionally undergo eruptions. The process of eruption of these prominences is caused by eruption of a huge magnetic system (HMS) associated with the prominence channel and the prominence itself (Rompolt 1984). Material of these prominences is frozen-into only a part of such a magnetic system before as well as during the eruption. When an HMS erupts it simply lifts with it the frozen-in prominence material.

Usually during the eruption a large part of the prominence material is lifted high into the corona and/or into interplanetary space. Nevertheless, on some occasions during the eruption a significant part of the original prominence material flows down to the chromosphere (Rompolt 1990).

Eruption of the QPs and ARPs in more or less the vertical direction proceeds with increasing speed from several kilometers per second at the beginning phase of the eruption up to several hundreds of kilometers per second at the late phase of the eruption. A typical eruptive prominence [EP] of type I (classification according to Rompolt 1984, 1990) is shown in Figure 1a, and the corresponding typical height-time $[\mathrm{H}-\mathrm{T}]$ curve one can find is shown in Figure $1 \mathrm{~b}$.

\section{Horizontal Expansion}

Besides the eruption in the vertical direction, some QPs as well as ARPs exhibit expansion in the horizontal direction with an average constant velocity of several tens of kilometers per second (Rompolt 1984). One classic example of such a horizontal expansion during the eruption of a QP on September 20, 1980 is shown in Figure 2. As the eruption proceeded, the southern extremity of the prominence was seen to be displaced more and more toward the south pole.

White arrows in Figure 2 in the panels labelled 08:07:01 UT and 10:36:12 (left) indicate the southern extremity of the prominence just before the eruption, whereas the right arrow in panel 10:36:12 UT shows the prominence's southern extremity at the end phase of the eruption in the light of the hydrogen $\mathrm{H} \alpha$ line. During the eruption the southern extremity of the prominence was displaced by $120,000 \mathrm{~km}$ with a constant velocity of $65 \mathrm{~km} / \mathrm{s}$. The displacement-time diagram [D-T] for five eruptive prominences showing horizontal expansion during their eruption is presented in Figure 3. Identify in this diagram the D-T dependence for the EP of September 20,1980 and compare it with it's H-T dependence shown in Figure 1b. 

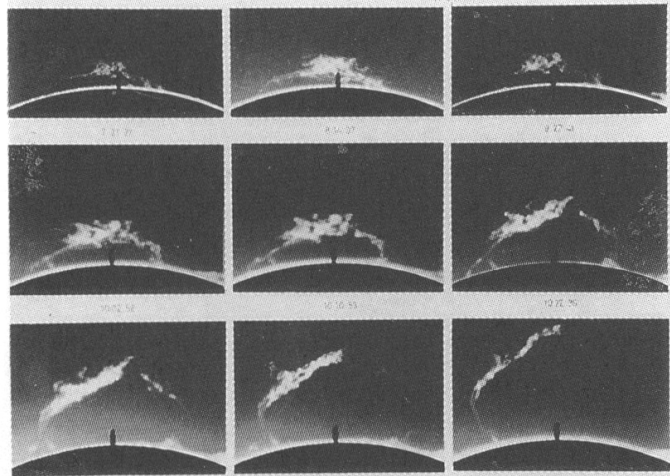

a

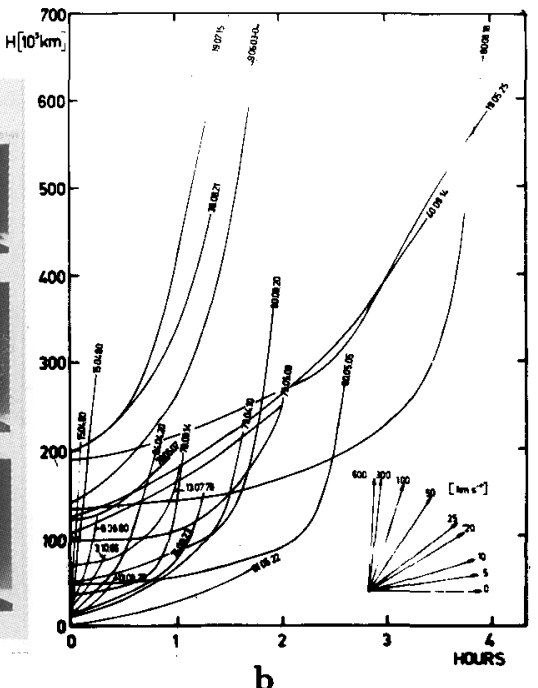

b

Figure 1. a: A typical example of an eruptive prominence [EP] of type I (taken with the SC), b: The height-time diagram for EPs - the zero level has been placed at the top of every prominence just before the eruption.
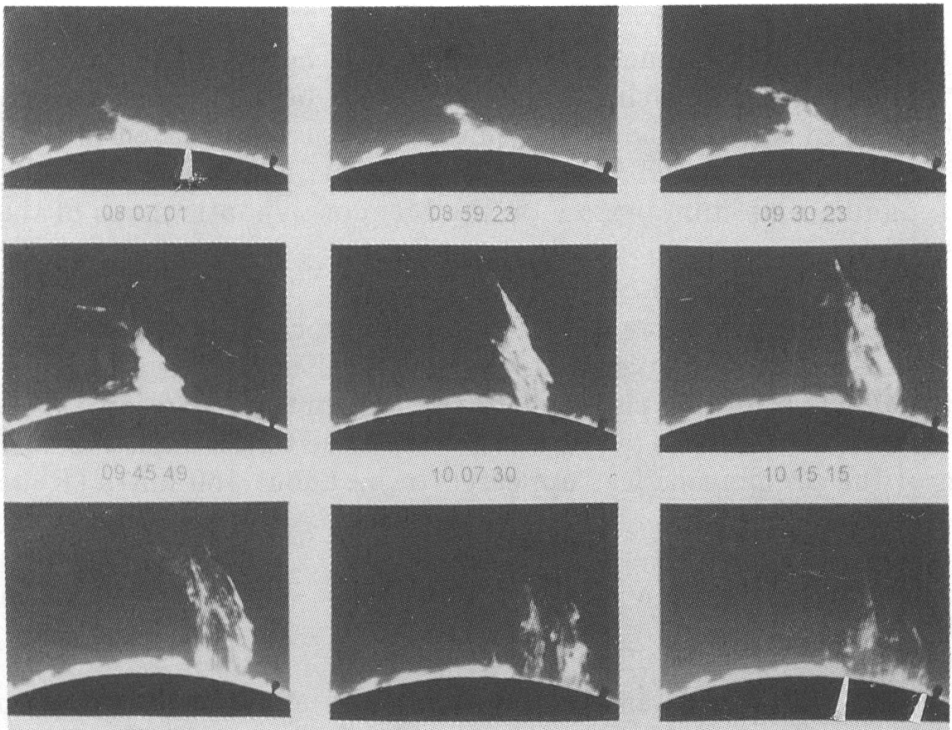

Figure 2. An eruptive prominence [EP] of type II on September 20, 1980 exhibiting during the eruption expansion in the horizontal direction (SC). 


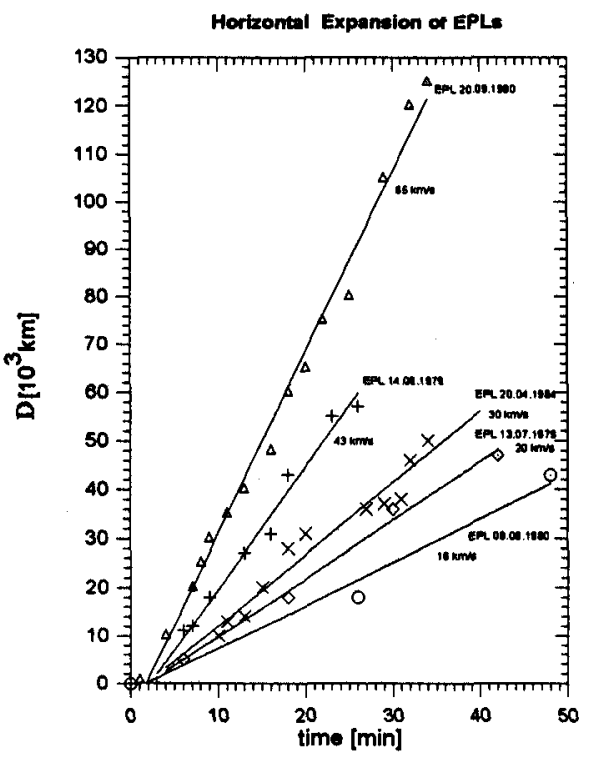

Figure 3. The horizontal displacement-time diagram [D-T] for five eruptive prominences showing horizontal expansion.

In deriving the displacements and velocities of horizontal expansion of the eruptive prominences, the inclination of the main body of these prominences towards the line of sight, while in the quiescent phase, was taken into account. This inclination was measured for these $\mathrm{H} \alpha$ filaments which were identified with the corresponding limb prominences on the Meudon synoptic maps of the chromosphere.

Horizontal expansion has been measured in a dozen or so EPs observed mainly with the Small Coronagraph (SC) of the Wroctaw Observatory. According to our measurements the velocities of horizontal expansion are in the range from 15 to $80 \mathrm{~km} / \mathrm{s}$. In one case, where the horizontal expansion was seen to spread out from the limb to a region on the disk close to the limb, the horizontal expansion of the prominence legs appeared to be about $160 \mathrm{~km} / \mathrm{s}$ (Rudawy et al. 1994)!

\section{Conclusion}

The horizontal expansion of an EP seems to be an effect of the eruption from the Sun of an associated HMS. In the course of this process the magnetic field is pulled out from the solar near-surface layers high into the corona and interplanetary space. In a later phase of the eruption when the bulk of the prominence material is lifted high into the corona, that part of the prominence material which flows down to the chromosphere is very likely gradually intercepted by a set of the prominence magnetic filaments being stretched out into the corona. 
These magnetic filaments, not visible during the earlier phase of eruption, now become visible in $\mathrm{H} \alpha$ due to the intercepted prominence material being subsequently cooled. This mechanism is schematically explained in Figure 4.

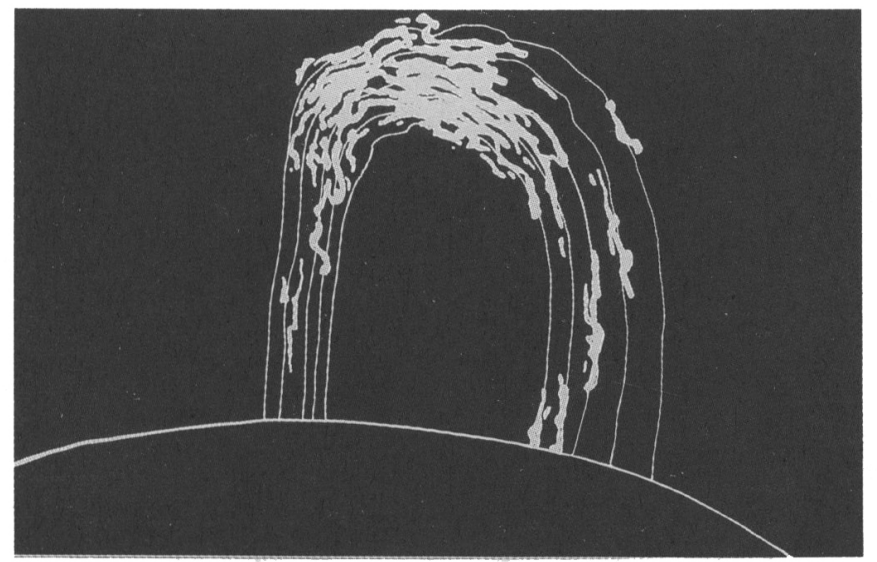

Figure 4. A drawing illustrating the mechanism of EPs' horizontal expansion.

The QPs as well as ARPs are formed by a system of intertwisted and/or interweaved fine filaments. During an eruption these prominences undergo untwisting into a system of fine loops (Rompolt 1984, 1990). In some cases, especially during a late phase of an eruption of the large, old QPs, one can identify several tens of fine loops.

Acknowledgments. This work has been supported by the Polish Committee for Scientific Researches under the project No. 776/P03/95/09. The author acknowledge the financial support allocated to him by the organizers.

\section{References}

Rompolt, B. 1984, Adv. Space Res., 4, No. 4, 357

Rompolt, B. 1990, Hvar Obs. Bull., 14, 37

Rudawy, P., Rompolt, B., Kotrč, P., Heinzel, P. and Knižek, M. 1994, in Solar Magnetic Fields, (eds.) M. Schlüsser and W. Schmidt, Cambridge University Press, p. 372 